\title{
Decreased FOXP3+ and GARP+Tregs to neoadjuvant chemotherapy associated with favorable prognosis in advanced gastric cancer
}

This article was published in the following Dove Press journal:

OncoTargets and Therapy

14 June 2016

Number of times this article has been viewed

\section{Kai $\mathrm{Li}^{\prime}$}

Fuchao Chen ${ }^{2}$

Huijuan $\mathrm{Xie}^{3}$

'Department of Pathology, ${ }^{2}$ Department of Clinical Pharmacy, ${ }^{3}$ Department of Hyperbaric Oxygen, Dongfeng Hospital, Hubei University of Medicine, Shiyan, People's Republic of China
Correspondence: Huijuan Xie Department of Hyperbaric Oxygen, Dongfeng Hospital, Hubei University of Medicine, 16 Daling Road, Zhangwan

District, Shiyan, Hubei Province,

People's Republic of China

Tel +860719 8268342

Email201220303000I@whu.edu.cn
Abstract: Neoadjuvant chemotherapy (NACT) has been an increasingly used therapeutic strategy to improve the outcome of advanced gastric cancer (GC) over the past few decades. Lymphocytic infiltration has been reported to be associated with response to NACT, but the immune cell subpopulation and its prognosis contributing to response in GC have not been clarified yet. In the current study, the tumor infiltration of FOXP3+ and GARP+ regulatory T-cells (Tregs, marked by FOXP3 and GARP) response to NACT in advanced GC and their correlation with prognosis were evaluated. The infiltration of FOXP3+ and GARP+ Tregs in 102 patients with advanced GC who were treated with or without NACT was measured using immunohistochemical method. The infiltration of FOXP3+ and GARP+ Tregs was significantly decreased in the NACT group than in the non-NACT group ( $P=0.023$ and $P=0.012$, respectively) and significantly associated with tumor, node, metastasis stage $(P=0.019$ and $P=0.011$, respectively). There was no significant difference in patient's overall survival between the NACT and non-NACT groups $(P=0.166)$; however, patients in the NACT group with decreased infiltration of FOXP3+ and GARP+ Tregs had longer overall survival $(P=0.002$ and $P<0.001$, respectively). Univariate and multivariate analyses indicated that the infiltration of GARP+ Tregs and lymph node metastasis were independent prognostic factors $(P=0.038$ and $P=0.013$, respectively). The results demonstrated that NACT could decrease the infiltration of FOXP3+ and GARP+ Tregs, and that the infiltration of GARP+ Tregs may serve as a new prognostic factor of human GC response to NACT.

Keywords: neoadjuvant chemotherapy, gastric cancer, Tregs, FOXP3, GARP

\section{Introduction}

Gastric cancer (GC) is the fourth most widespread tumor in the world and the second most common cause of cancer-related deaths worldwide currently, regardless of its decreased incidence. ${ }^{1}$ Generally, survival rates for this malignancy are low, because in most cases, patients with GC are diagnosed at advanced stage, with local extension or lymph node metastasis, when the treatment is unlikely to succeed. ${ }^{2}$ Surgery remains the only potentially curative treatment for this disease in operable stages, ${ }^{3}$ while chemotherapy for advanced GC could play an important role in improving the prognosis of the patients. ${ }^{4}$

Neoadjuvant chemotherapy (NACT) has been investigated with different protocols in order to prevent the local relapse and improve survival time of patients with advanced GC for decades. Some studies indicated that preoperative chemotherapy is potentially down-staging the tumor stages and improves the survival rates of patients with advanced GC, while some studies suggested that this treatment protocol was 
hazardous. ${ }^{5,6}$ Recently, lymphocytic infiltration associated with response to NACT has been proposed, but the immune cell subpopulation and its prognosis contributing to response have not been clarified yet. Thus, studies should be investigated to explore the immune cell subpopulations as predictive factors that affect treatment response to NACT.

The role of immune response in GC is not fully understood, but recent observations suggest that chemotherapy may trigger an immune response, which may contribute to treatment response. ${ }^{7}$ Previous studies suggested that tumorinfiltrating lymphocytes (TILs) are deemed to play a vital role in the immune response against malignant tumors and the prognostic implications of TILs in GC depend on the density and type of TILs. ${ }^{8-10}$ Regulatory T-cells (Tregs) are a kind of immunosuppressive T-lymphocytes that play a major role in immune escape and suppressing antitumor immune response, thus promoting tumor growth and invasion. ${ }^{11,12}$ FOXP3, a transcription factor that regulates the development of Tregs, is considered to be a marker of Tregs. However, recent studies indicated that FOXP3 cannot be used as a reliable marker for Tregs since effector T-cells also upregulate FOXP3 expression upon activation. ${ }^{13}$ GARP is selectively expressed only in activated human Tregs but not in activated effector T-cells, confirming GARP to be a bona fide Treg marker. ${ }^{14}$ Herein, both FOXP3 and GARP were considered as the markers of Tregs.

The effect of NACT on tumor-infiltrating immune cells and their change predict the response and prognosis of breast cancer, ${ }^{15}$ and intratumoral Tregs have been reported in breast cancer with positive correlation between the density of Tregs and prognosis; ${ }^{16}$ however, such report on GC has been limited.

The aim of this study was to investigate the predictive and prognostic value of FOXP3 + and GARP+ Tregs in advanced GC. The number of FOXP3+ and GARP+ Tregs (marked by FOXP3 and GARP) in patients with advanced GC who were treated with or without NACT was evaluated. Then, the correlation of FOXP3+ and GARP+ Tregs with prognosis and the overall survival (OS) of patients with GC who were treated with NACT were analyzed.

\section{Patients and methods}

\section{Patients}

A total of 102 patients with GC who received surgical treatment between 2008 and 2013 in Dongfeng Hospital, Hubei University of Medicine, Shiyan, People's Republic of China, were retrospectively evaluated. Fifty-six patients received NACT before surgery, whereas the remaining 46 patients received surgical treatment without NACT. The clinicopathologic data, such as time of cancer diagnosis, chemotherapy protocol, sex, age, clinical stage of carcinoma, histological type of tumor, follow-up procedures, and patient's OS, were obtained from the Department of Oncology, Dongfeng Hospital, Hubei University of Medicine (Table 1). The tumor, lymph node, metastasis (TNM) staging of GC was determined according to the American Joint Committee on Cancer. ${ }^{17}$ Follow-up duration was defined as the time (months) from the diagnosis of GC till the final visit. The OS was defined as the time from the diagnosis of GC till the patient's death (due to any cause) or the final visit. The written informed consent for the tissue ex vivo experimentation was obtained from all the patients. The ethics committee of Dongfeng Hospital, Hubei University of Medicine has approved this study (scientific research ethics reference number 2013002).

The detailed FOLFOX6 chemotherapy protocol was as follows: patients received oxaliplatin at $85 \mathrm{mg} / \mathrm{m}^{2}$ (intravenous [IV] injection, $>2$ hours), calcium folinate at $400 \mathrm{mg} / \mathrm{m}^{2}$

Table I Demographics and clinicopathologic characteristics of patients

\begin{tabular}{|c|c|c|c|}
\hline & $\begin{array}{l}\text { NACT } \\
\text { group } \\
(n=56)\end{array}$ & $\begin{array}{l}\text { non-NACT } \\
\text { group } \\
(n=46)\end{array}$ & $P$-value \\
\hline \multicolumn{4}{|l|}{ Sex, n (\%) } \\
\hline Male & $36(64.3)$ & $26(56.5)$ & 0.424 \\
\hline Female & $20(35.7)$ & $20(43.5)$ & \\
\hline \multicolumn{4}{|l|}{ Age (years), n (\%) } \\
\hline$<55$ & $20(35.7)$ & $22(47.8)$ & 0.216 \\
\hline$\geq 55$ & $36(64.3)$ & $24(52.2)$ & \\
\hline \multicolumn{4}{|l|}{ Differentiation, n (\%) } \\
\hline Well/moderately & $20(35.7)$ & $12(26.1)$ & 0.299 \\
\hline Poorly & $36(64.3)$ & 34 (73.9) & \\
\hline \multicolumn{4}{|l|}{ Invasion depth, n (\%) } \\
\hline $\mathrm{T} 2$ & $16(28.6)$ & $10(21.7)$ & 0.433 \\
\hline $\mathrm{T} 3 / \mathrm{T} 4$ & $40(71.4)$ & $36(78.3)$ & \\
\hline \multicolumn{4}{|l|}{ TNM stage, $\mathrm{n}(\%)$} \\
\hline II & $24(42.9)$ & $14(30.4)$ & 0.199 \\
\hline III + IV & $32(57.1)$ & $32(69.6)$ & \\
\hline \multicolumn{4}{|l|}{ Lymph node metastasis, $\mathrm{n}(\%)$} \\
\hline No (N0) & $28(50.0)$ & $16(34.8)$ & 0.123 \\
\hline Yes (NI, N2, and N3) & $28(50.0)$ & $30(65.2)$ & \\
\hline \multicolumn{4}{|l|}{ Clinical status at follow-up, n (\%) } \\
\hline Dead or died with recurrence & $28(50.0)$ & $30(65.2)$ & - \\
\hline Live without recurrence & $28(50.0)$ & $16(34.8)$ & \\
\hline \multicolumn{4}{|l|}{ FOXP3+ Tregs } \\
\hline Low, n (\%) & $37(66.1)$ & $20(43.5)$ & 0.023 \\
\hline High, n (\%) & $19(33.9)$ & $26(56.5)$ & \\
\hline \multicolumn{4}{|l|}{ GARP+ Tregs } \\
\hline Low, n (\%) & $36(64.3)$ & $18(39.1)$ & 0.012 \\
\hline High, n (\%) & $20(35.7)$ & $28(60.9)$ & \\
\hline
\end{tabular}

Notes: T2, tumor invasion of muscularis propria; T3, tumor invasion of the subserosal connective tissue; T4, tumor invasion of serosal or adjacent structures; well/ moderately, well/moderately differentiated carcinoma; poorly, poorly differentiated carcinoma.

Abbreviations: NACT, neoadjuvant chemotherapy; TNM, tumor, node, metastasis; Tregs, regulatory T-cells. 
(IV $>2$ hours), and 5-fluorouracil $500 \mathrm{mg}$, (IV by continuous infusion for 44 hours). Patients received two cycles of chemotherapy, with each cycle of 3 weeks.

\section{Immunohistochemical staining}

Conventional streptavidin peroxidase (S-P) immunohistochemical (IHC) staining protocol was used in this study. Briefly, paraffin-embedded tumor tissue blocks were cut into species of $4 \mu \mathrm{m}$ thickness, and then they were dried, deparaffinized, and dehydrated. Tissue sections were treated with $1 \%$ hydrogen peroxide for 10 minutes to block endogenous tissue peroxidase, followed by treatment with bovine serum for 30 minutes to reduce nonspecific binding. Then, antigen retrieval using citrate buffer ( $\mathrm{pH}$ 6.0) was accomplished as follows: high heat microwave processing for 5 minutes followed by low heat microwave processing for 20 minutes. All the slides were incubated with primary rabbit antihuman FOXP3 polyclonal antibody (BA2032-1, 1:200 dilution, marker for Tregs; Wuhan Boster Biological Technology, Ltd., Wuhan, People's Republic of China) and mouse antihuman GARP monoclonal antibody (S1/61/69, 1:100 dilution, marker for Tregs; Zhongshan Golden Bridge Biotechnology, Ltd., Beijing, People's Republic of China) for 1 hour at $37^{\circ} \mathrm{C}$ and $4{ }^{\circ} \mathrm{C}$ overnight, respectively, followed by a 30-minute incubation in UltraSensitive SP Kit (Maixin-Bio, Fuzhou, People's Republic of China). All slides were rinsed with phosphate-buffered saline, color was developed using 3,3'-diaminobenzidine substrate kit, and then the slides were counterstained with hematoxylin.

The slides were examined under Olympus BX53 microscope (Olympus China Co. Limited, Beijing, People's Republic of China) by two senior pathologists who were blinded to the clinicopathologic data. In the case of discrepancy, a consensus was achieved using a multiheaded microscope. Cytoplasmic staining with FOXP3 and GARP antibodies in lymphocytes was defined as positive. IHC staining of FOXP3 and GARP proteins was assessed in terms of staining intensity and percentage of positive cells as follows: 0 (negative), 1+ (weak staining, $\leq 10 \%$ of cells staining positive), $2+$ (moderate staining, $10 \%-90 \%$ of cells staining positive), and $3+$ (strong staining, $>90 \%$ of cells staining positive). The final score for each slide was represented by the average of three representative high-power fields $(\times 400)$. Scores $\leq 1+$ were defined as low expression, and scores $\geq 2+$ were defined as high expression.

\section{Statistical analysis}

Statistical analysis was performed using SPSS 17.0 (IBM company, Armonk, NY, USA). The differences of FOXP3+, GARP+ Tregs, and clinicopathologic parameters between two groups were analyzed using chi-square test. Spearman's correlation analysis was used to determine the correlation between clinicopathologic features and FOXP3+ and GARP+ Tregs in the NACT group. The OS was analyzed using Kaplan-Meier method and Cox proportional hazards regression method. All two-sided $P$-values $<0.05$ were considered statistically significant.

\section{Results}

\section{Patient characteristics and IHC features}

The median follow-up duration was 24 months (range: 1-58 months). There were 62 male and 40 female patients; median age at surgery was 55 years (range: 30-78 years). The patients were divided into two groups: patients who received NACT before surgery (NACT group, $n=56$ ) and who did not receive NACT treatment before surgery (nonNACT group, $n=46$ ). The patient's characteristics are shown in Table 1. The association of FOXP3+ and GARP+ Tregs with clinicopathological features of advanced GC treated with or without NACT was evaluated. It was found that the infiltration of FOXP3+ and GARP+ Tregs in the NACT group was significantly decreased compared to that in the non-NACT group ( $P=0.023$ and $P=0.012$, respectively). There were no clinicopathological parameters associated with NACT, including sex $(P=0.424)$, age $(P=0.216)$, differentiation $(P=0.299)$, TNM stage $(P=0.199)$, invasion depth $(P=0.433)$, and lymph node status $(P=0.123)$.

Positive nuclear staining with FOXP3 and GARP antibodies in intratumoral tissues was considered FOXP3+ and GARP+ Tregs. In the NACT group, 37 (66.1\%) and 36 (64.3\%) patients showed no or low infiltration of FOXP3+ and GARP+ Tregs, respectively, and 19 (33.9\%) and 20 (35.7\%) patients showed high infiltration of FOXP3+ and GARP+ Tregs, respectively. While in patients with GC in the non-NACT group, $20(43.5 \%)$ and $18(39.1 \%)$ patients showed no or low infiltration of FOXP3 + and GARP+ Tregs, respectively, and the other $26(56.5 \%)$ and $28(60.9 \%)$ patients showed high infiltration of FOXP3+ and GARP+ Tregs, respectively. The infiltration of FOXP3 + and GARP+ Tregs in the NACT group was less than that in the non-NACT group (Figure 1).

\section{Association between FOXP3 and GARP expressions with clinicopathological parameters in the NACT group}

Spearman's correlation analysis showed that the infiltration of FOXP3+ and GARP+ Tregs in the NACT group was not correlated with clinicopathologic features (all $P>0.05$ ) except TNM stage, where I+ II had lower infiltration of FOXP $3+$ and GARP+ Tregs $(P=0.019$ and $P=0.011$, respectively; Table 2$)$. The infiltration of FOXP3 + Treg was significantly consistent with the infiltration of GARP+ Treg $(P<0.001)$. 

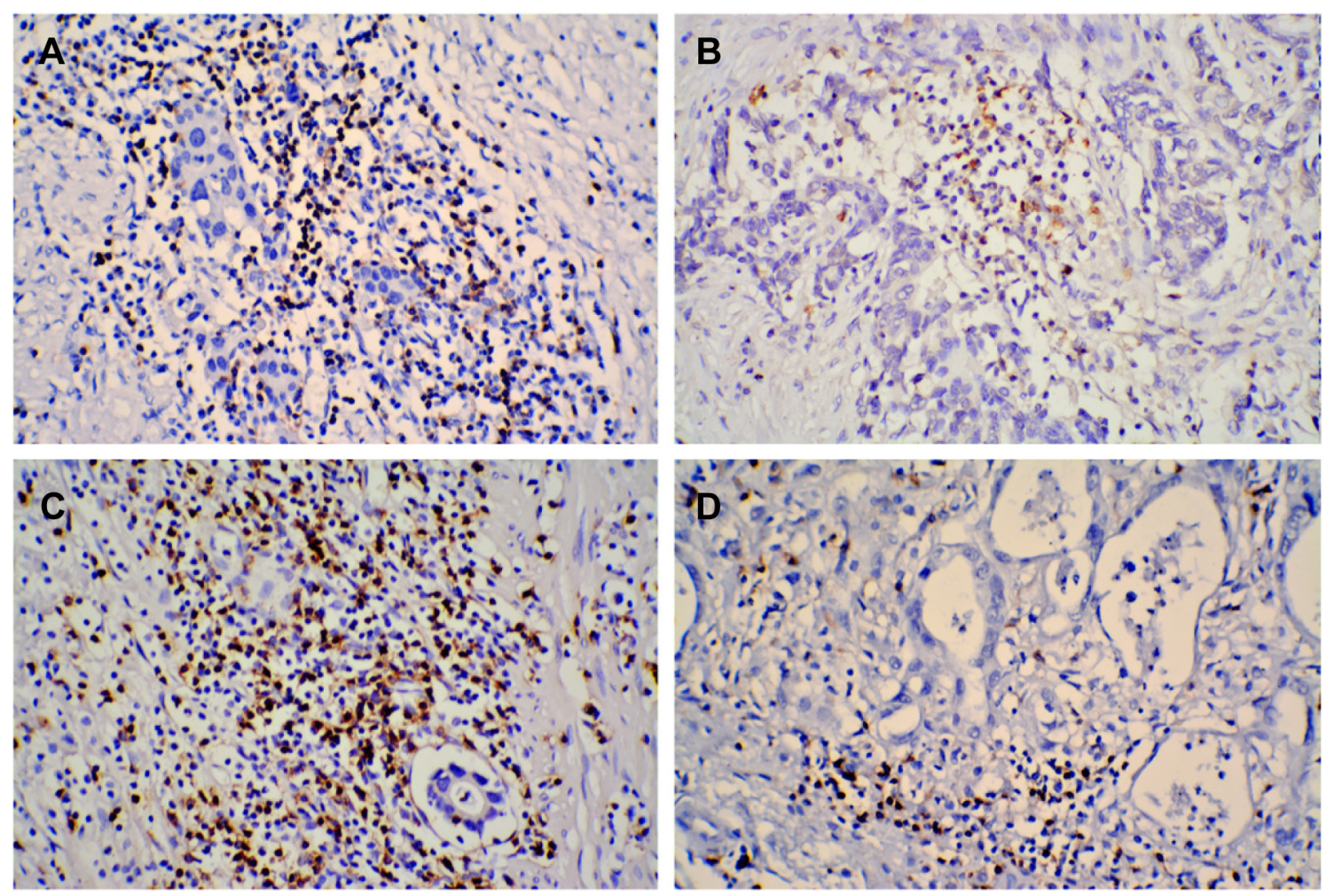

Figure I IHC staining of FOXP3 and GARP proteins was mainly observed in intratumoral tissues of GC.

Notes: The infiltration of FOXP3+ (A) and GARP+ (C) Tregs was higher in the non-NACT group than the infiltration of FOXP3+ (B) and GARP+ (D) Tregs in the NACT group (original magnification, $\times 200$ ).

Abbreviations: GC, gastric cancer; IHC, immunohistochemical; NACT, neoadjuvant chemotherapy; Tregs, regulatory T-cells.

Table 2 Relationship between FOXP3 and GARP expressions and clinicopathologic parameters in the NACT group

\begin{tabular}{|c|c|c|c|c|c|c|}
\hline & \multicolumn{2}{|c|}{ FOXP3+ Tregs } & \multirow[t]{2}{*}{$P$-value } & \multicolumn{2}{|c|}{ GARP+ Tregs } & \multirow[t]{2}{*}{$P$-value } \\
\hline & Low $(n=37)$ & High $(n=19)$ & & Low $(n=36)$ & High $(n=20)$ & \\
\hline \multicolumn{7}{|l|}{ Sex } \\
\hline Male & 26 & 10 & 0.196 & 24 & 12 & 0.621 \\
\hline Female & II & 9 & & 12 & 8 & \\
\hline \multicolumn{7}{|l|}{ Age (years) } \\
\hline$<55$ & 13 & 7 & 0.900 & 12 & 8 & 0.621 \\
\hline$\geq 55$ & 24 & 12 & & 24 & 12 & \\
\hline \multicolumn{7}{|l|}{ Differentiation } \\
\hline Well/moderately & 16 & 4 & 0.104 & 12 & 8 & 0.621 \\
\hline Poorly & 21 & 15 & & 24 & 12 & \\
\hline \multicolumn{7}{|l|}{ Invasion depth } \\
\hline $\mathrm{T} 2$ & 12 & 4 & 0.376 & 12 & 4 & 0.294 \\
\hline $\mathrm{T} 3 / \mathrm{T} 4$ & 25 & 15 & & 24 & 16 & \\
\hline \multicolumn{7}{|l|}{ TNM stage } \\
\hline II & 20 & 4 & 0.019 & 20 & 4 & 0.011 \\
\hline$I I I+I V$ & 17 & 15 & & 16 & 16 & \\
\hline \multicolumn{7}{|c|}{ Lymph node metastasis } \\
\hline No & 20 & 8 & 0.401 & 20 & 8 & 0.269 \\
\hline Yes & 17 & 11 & & 16 & 12 & \\
\hline \multicolumn{7}{|l|}{ FOXP3+ Tregs } \\
\hline Low & NA & NA & NA & 33 & 4 & 0.000 \\
\hline High & NA & NA & & 3 & 16 & \\
\hline \multicolumn{7}{|l|}{ GARP+ Tregs } \\
\hline Low & 33 & 3 & 0.000 & NA & NA & NA \\
\hline High & 4 & 16 & & NA & NA & \\
\hline
\end{tabular}

Notes: T2, tumor invasion of muscularis propria; T3, tumor invasion of subserosal connective tissue; T4, tumor invasion of serosal or adjacent structures; well/moderately, well/moderately differentiated carcinoma; poorly, poorly differentiated carcinoma.

Abbreviations: NACT, neoadjuvant chemotherapy; TNM, tumor, node, metastasis; Tregs, regulatory T-cells; NA, no data. 


\section{Association between OS and FOXP3 as well as GARP expression responses to NACT}

The median survival time of patients who were treated with or without NACT was 23.5 months (range: 10-58 months) and 26 months (range: 1-56 months). As per univariate analysis, there was no significant difference between OS of both the NACT and non-NACT groups (log-rank test, $P=0.166$; Figure S1). In the NACT group, patients with a lower infiltration of FOXP3+ Tregs had a longer OS than patients with a higher infiltration of FOXP3+, while in non-NACT group,
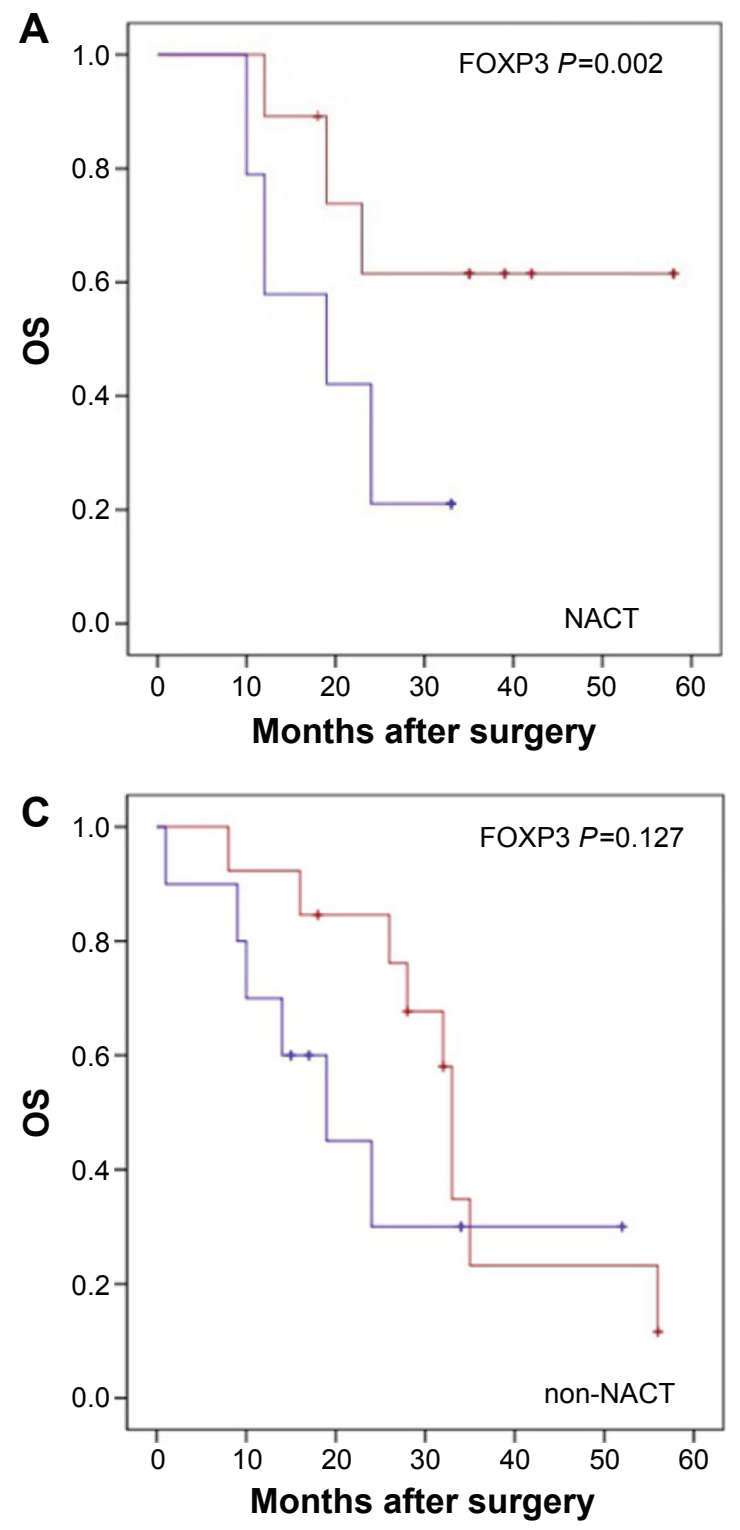

there was no correlation between patient's OS and the infiltration of FOXP3+ Tregs (log-rank test, $P=0.002$ and $P=0.127$, respectively; Figure $2 \mathrm{~A}$ and $\mathrm{B})$. Similarly, patients with a lower infiltration of GARP+ Tregs in the NACT group had a longer OS than patients with a higher infiltration of GARP+ Tregs, while in the non-NACT group, there was no correlation between patient's OS and the infiltration of GARP+ Tregs (log-rank test, $P<0.001$ and $P=0.269$, respectively; Figure 2C and D). The OS in the NACT group was also significantly associated with TNM stage (log-rank test, $P=0.037$; Figure S2).
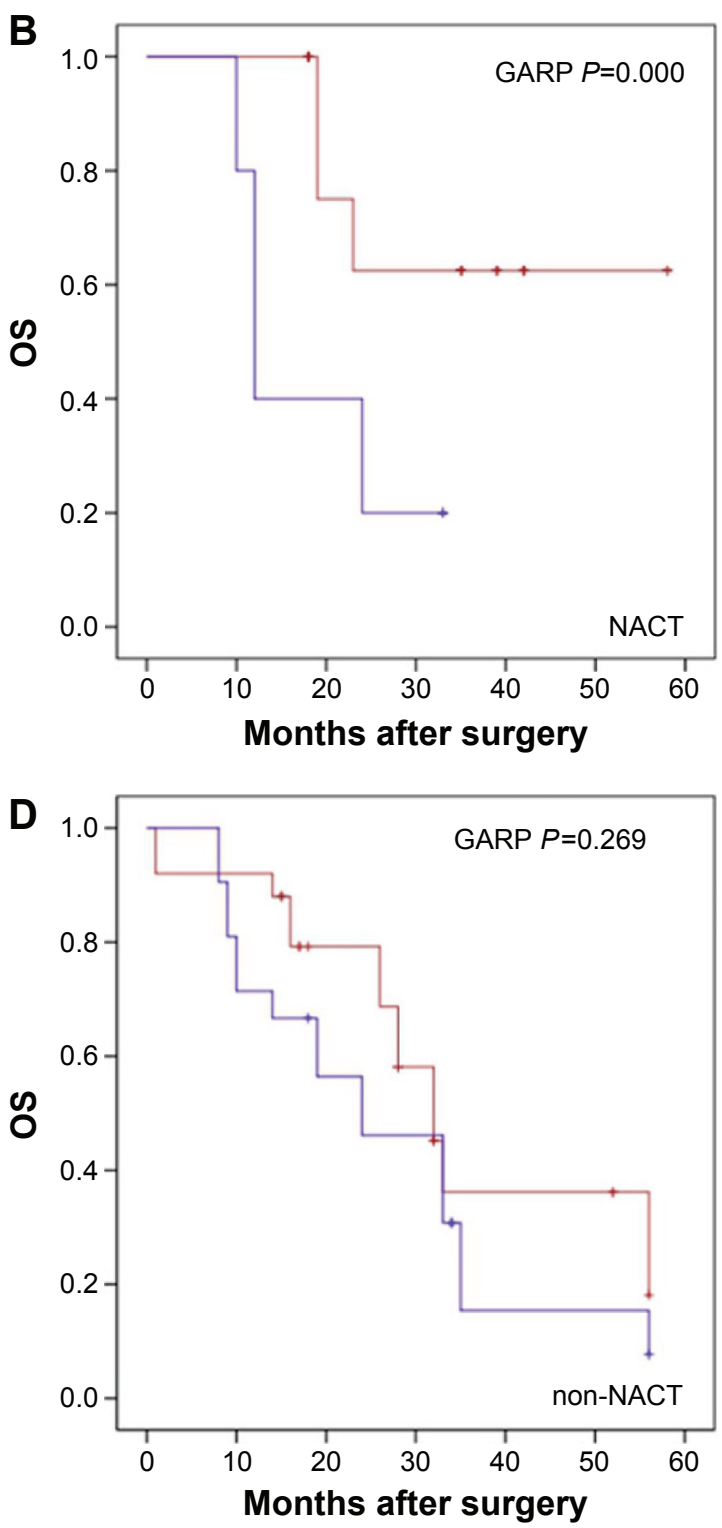

$\neg$ Low expression + Low expression censored $\neg$ High expression + High expression censored

Figure 2 Kaplan-Meier analysis of OS.

Notes: (A) Patients in the NACT group with decreased infiltration of FOXP3+ Tregs had longer OS (log-rank test, $P=0.002$ ); (B) No significant difference was seen between the infiltration of FOXP3+ Tregs in patients in the non-NACT group (log-rank test, $P=0.127$ ). (C) Patients in the NACT group with decreased infiltration of GARP+ Tregs had longer OS (log-rank test, $P<0.001$ ); (D) No significant difference was seen between the infiltration of GARP+ Tregs in patients in the non-NACT group (log-rank test, $P=0.269$ ). Abbreviations: NACT, neoadjuvant chemotherapy; OS, overall survival; Tregs, regulatory T-cells. 
The factors found to have an effect on OS in the NACT group were reanalyzed using Cox proportional hazards regression method (Table 3). Among the variables, the infiltration of GARP+ Tregs and lymph node metastasis were two effective independent factors for OS. Patients with GC with higher infiltration of GARP+ Tregs and with lymph node metastasis had an increased risk of death $(P=0.013$ and $P=0.038$, respectively).

\section{Discussion}

NACT has been increasingly used to improve the curative surgical resection and decrease the risk of metastasis, thus prolonging the survival time of patients with advanced GC over the past decades. ${ }^{6}$ However, the efficacy of NACT is difficult to evaluate because the clinical responses predicting the prognostic significance of NACT vary and result in disparate outcomes..$^{18}$

TILs in the tumor microenvironment have shown the prognostic value in large clinical series of cancer, but the contribution to the clinical and biological behavior of the tumor depends on the TIL subtypes and density. ${ }^{8-10}$ Tregs, known as immunosuppressive T-lymphocytes, play a major role in

Table 3 Predictive factors for long-term survival by univariate and multivariate analysis in the NACT group

\begin{tabular}{|c|c|c|c|c|}
\hline & $\mathbf{n}$ & $\begin{array}{l}\text { Log-rank test } \\
\chi^{2} \text { value }\end{array}$ & $\begin{array}{l}P \text {-value } \\
\text { univariate }\end{array}$ & $\begin{array}{l}P \text {-value } \\
\text { multivariate }\end{array}$ \\
\hline \multicolumn{5}{|l|}{ Age (years) } \\
\hline$<55$ & 20 & I.47I & 0.225 & 0.058 \\
\hline$\geq 55$ & 36 & & & \\
\hline \multicolumn{5}{|l|}{ Differentiation } \\
\hline Well/moderately & 20 & 0.803 & 0.370 & 0.796 \\
\hline Poorly & 36 & & & \\
\hline \multicolumn{5}{|l|}{ Invasion depth } \\
\hline $\mathrm{T} 2$ & 16 & 3.424 & 0.064 & 0.162 \\
\hline $\mathrm{T} 3 / \mathrm{T} 4$ & 40 & & & \\
\hline \multicolumn{5}{|l|}{ TNM stage } \\
\hline ॥ & 24 & 4.330 & 0.037 & 0.137 \\
\hline $\mathrm{III}+\mathrm{IV}$ & 32 & & & \\
\hline \multicolumn{5}{|c|}{ Lymph node metastasis } \\
\hline No & 28 & 2.546 & 0.111 & 0.038 \\
\hline Yes & 28 & & & \\
\hline \multicolumn{5}{|l|}{ FOXP3+ Tregs } \\
\hline High & 19 & 9.825 & 0.002 & 0.652 \\
\hline Low & 37 & & & \\
\hline \multicolumn{5}{|l|}{ GARP+ Tregs } \\
\hline High & 20 & 14.610 & 0.000 & 0.013 \\
\hline Low & 36 & & & \\
\hline
\end{tabular}

Notes: T2, tumor invasion of muscularis propria; $\mathrm{T} 3$, tumor invasion of subserosal connective tissue; T4, tumor invasion of serosal or adjacent structures; well/ moderately, well/moderately differentiated carcinoma; poorly, poorly differentiated carcinoma.

Abbreviations: NACT, neoadjuvant chemotherapy; TNM, tumor, node, metastasis; Tregs, regulatory T-cells. immune escape and suppressing antitumor immune response, thus promoting tumor growth and invasion. Previous studies have indicated that TILs are the potent predictive factors for response to NACT in breast cancer, and intratumoral Tregs have been reported with positive correlation between the density of Tregs and prognosis in breast cancer, ${ }^{19,20}$ but the effects of NACT on GC immune response are limited.

Herein, our current study demonstrated that NACT treatment induced significant changes in the infiltration of FOXP3+ and GARP+ Tregs resulted in a prolonged OS. First, we evaluated intratumoral infiltration of FOXP3+ and GARP+ Tregs in both the groups of patients, and then correlation between the changes of FOXP3+ and GARP+ Tregs in the NACT group and clinicopathological features as well as the prognosis of GC were evaluated. NACT could decrease the infiltration of FOXP3 + and GARP+ Tregs in tumor microenvironment, and the changes were positively correlated with clinicopathological parameters and prolonged OS of advanced GC. In addition, our results indicated that the infiltration of GARP+ Tregs could be served as an independent prognostic factor, which is the predictive of response to NACT.

Recently, the role of NACT in advanced GC is controversial because of diverse preoperative clinical and pathological response to NACT. ${ }^{21}$ Accordingly, in the current study, figuring out the potential predictive factors that represent response to NACT is of great importance. This study demonstrated that NACT decreased FOXP3+ and GARP+ Tregs in tumor stroma resulted in prolonged OS. Thus, we hypothesized that these clinical results may be partly associated with an enhanced tumor-specific immunity related to NACT, and the immunity of the patients with GC might be a predictive factor of response to NACT.

It is well known that FOXP3+ Tregs are a kind of immunosuppressive T-lymphocytes, and it was demonstrated that the accumulation of FOXP3+ Tregs around tumor tissues could promote tumor growth or distinct metastasis by means of its immunosuppressive function in many cancers. Previous studies have indicated that Tregs are sensitive to chemotherapy, and the antitumor activity of chemotherapy is mediated by depletion and suppression of Tregs and lower number of intratumoral FOXP3+ Tregs with NACT with the favorable prognostic effect in breast cancer. ${ }^{22}$ However, subsequent studies found that FOXP3 was expressed in nonTregs and effector T-cells except for Tregs, and FOXP3 was an intracellular marker that did not allow Treg isolation. ${ }^{23,24}$ Therefore, efforts should be made into identifying effective markers for Tregs. 
GARP is a newly identified molecule on Tregs, which is the receptor of latent transforming growth factor $\beta$ of activated Treg, and is highly expressed in activated FOXP3+ Tregs to promote immunosuppression. ${ }^{25}$ GARP efficiently represses the proliferation and differentiation of naive CD4+ T-cells into T-effector cells ${ }^{26}$ induces Foxp3, decreases proliferation, and represses interleukin- 2 and interferon- $\gamma$ productions, resulting in differentiation of naive T-cells into an induced $\mathrm{Treg}^{23}$ and demonstrating that GARP played a key role in Tregs maturation and function. In this study, the infiltration of GARP+ Tregs was decreased in patients with GC who were treated with NACT and resulted in a prolonged OS. What is more, GARP+ Tregs but not FOXP3+ Tregs were served as the independent prognostic factors in advanced GC, confirming that GARP+ Tregs are bona fide Tregs with immunosuppressive characteristics.

Overall, the results of the current study demonstrated that patients with advanced GC who were treated with NACT achieved a significantly decreased infiltration of FOXP3+ Tregs, especially GARP+ Tregs, which means that immune suppression of patients with advanced GC was significantly relieved after NACT. However, the current study had several limitations. There are only a small proportion of patients, and the heterogeneity of patients' response to NACT was not considered in this study, which could affect the clinical outcomes. The 5-year survival rates of the study group were not available to follow up; hence, it would be appropriate to repeat the OS analyses after 5 years of follow-up.

\section{Conclusion}

The current study indicated that NACT may be considered to have effects on antitumor immune response by decreasing intratumoral FOXP3+ and GARP+ Tregs, thus resulting in a favorable prognosis of advanced GC. Further more, it is the intratumoral infiltration of GARP+ Tregs, which represents the activated Tregs, but not FOXP3+ Tregs that could be an independent prognostic predictor response to NACT in advanced GC. However, the mechanisms leading to the decrease in the infiltration of FOXP3+ and GARP+ Tregs in tumor stroma by NACT and thus promoting antitumor immune response need to be investigated further.

\section{Disclosure}

The authors report no conflicts of interest in this work.

\section{References}

1. Ferlay J, Shin HR, Bray F, Forman D, Mathers C, Parkin DM. Estimates of worldwide burden of cancer in 2008: GLOBOCAN 2008. Int J Cancer. 2010;127(12):2893-2917.
2. Lochhead P, El-Omar EM. Gastric cancer. Br Med Bull. 2008;85: 87-100.

3. $\mathrm{Hu} \mathrm{JK}, \mathrm{Li} \mathrm{CM}$, Chen XZ, et al. The effectiveness of intravenous 5 -fluorouracil-containing chemotherapy after curative resection for gastric carcinoma: a systematic review of published randomized controlled trials. J Chemother. 2007;19(4):359-375.

4. Rivera F, Vega-Villegas ME, Lopez-Brea MF. Chemotherapy of advanced gastric cancer. Cancer Treat Rev. 2007;33(4):315-324.

5. Li ZY, Koh CE, Bu ZD, et al. Neoadjuvant chemotherapy with FOLFOX: improved outcomes in Chinese patients with locally advanced gastric cancer. J Surg Oncol. 2012;105(8):793-799.

6. An JY, Kim HI, Cheong JH, Hyung WJ, Kim CB, Noh SH. Pathologic and oncologic outcomes in locally advanced gastric cancer with neoadjuvant chemotherapy or chemoradiotherapy. Yonsei Med J. 2013;54(4): 888-894.

7. Zitvogel L, Kepp O, Kroemer G. Immune parameters affecting the efficacy of chemotherapeutic regimens. Nat Rev Clin Oncol. 2011;8(3): $151-160$.

8. Galon J, Costes A, Sanchez-Cabo F, et al. Type, density, and location of immune cells within human colorectal tumors predict clinical outcome. Science. 2006;313(5795):1960-1964.

9. Zhang L, Conejo-Garcia JR, Katsaros D, et al. Intratumoral T cells, recurrence, and survival in epithelial ovarian cancer. $N$ Engl J Med. 2003; 348(3):203-213.

10. Lee HE, Chae SW, Lee YJ, et al. Prognostic implications of type and density of tumour-infiltrating lymphocytes in gastric cancer. Br J Cancer. 2008;99(10):1704-1711.

11. Whiteside TL. What are regulatory $\mathrm{T}$ cells (Treg) regulating in cancer and why? Semin Cancer Biol. 2012;22(4):327-334.

12. Whiteside TL, Schuler $P$, Schilling B. Induced and natural regulatory T cells in human cancer. Expert Opin Biol Ther. 2012;12(10):1383-1397.

13. Probst-Kepper M, Buer J. FOXP3 and GARP (LRRC32): the master and its minion. Biol Direct. 2010;5:8.

14. Battaglia M, Roncarolo MG. The Tregs' world according to GARP. Eur J Immunol. 2009;39(12):3296-3300.

15. García-Martínez E, Gil GL, Benito AC, et al. Tumor-infiltrating immune cell profiles and their change after neoadjuvant chemotherapy predict response and prognosis of breast cancer. Breast Cancer Res. 2014; 16(6):488

16. Liu FF, Li YQ, Ren MJ, et al. Peritumoral FOXP3(+) regulatory T cell is sensitive to chemotherapy while intratumoral FOXP3 $(+)$ regulatory $\mathrm{T}$ cell is prognostic predictor of breast cancer patients. Breast Cancer Res Treat. 2012;135(2):459-467.

17. Washington K. 7th edition of the AJCC cancer staging manual: stomach. Ann Surg Oncol. 2010;17(12):3077-3079.

18. Lorenzen S, Blank S, Lordick F, Siewert JR, Ott K. Prediction of response and prognosis by a score including only pretherapeutic parameters in 410 neoadjuvant treated gastric cancer patients. Ann Surg Oncol. 2012;19(7):2119-2127.

19. Denkert C, Loibl S, Noske A, et al. Tumor-associated lymphocytes as an independent predictor of response to neoadjuvant chemotherapy in breast cancer. J Clin Oncol. 2010;28(1):105-113.

20. Ono M, Tsuda H, Shimizu C, et al. Tumor-infiltrating lymphocytes are correlated with response to neoadjuvant chemotherapy in triple-negative breast cancer. Breast Cancer Res Treat. 2012;132(3):793-805.

21. Ychou M, Boige V, Pignon JP, et al. Perioperative chemotherapy compared with surgery alone for resectable gastroesophageal adenocarcinoma: an FNCLCC and FFCD multicenter phase III trial. J Clin Oncol. 2011;29(13):1715-1721.

22. Ladoire S, Mignot G, Dabakuyo S, et al. In situ immune response after neoadjuvant chemotherapy for breast cancer predicts survival. $J$ Pathol. 2011;224(3):389-400

23. Elkord E, Abd Al Samid M, Chaudhary B. Helios, and not FoxP3, is the marker of activated Tregs expressing GARP/LAP. Oncotarget. 2015; 6(24):20026-20036. 
24. Abd Al Samid M, Chaudhary B, Khaled YS, Ammori BJ, Elkord E. Combining FoxP3 and Helios with GARP/LAP markers can identify expanded Treg subsets in cancer patients. Oncotarget. Epub 2016 Feb 11.

25. Gauthy E, Cuende J, Stockis J, et al. GARP is regulated by miRNAs and controls latent TGF-beta1 production by human regulatory $\mathrm{T}$ cells. PLoS One. 2013;8(9):e76186.
26. Hahn SA, Stahl HF, Becker C, et al. Soluble GARP has potent antiinflammatory and immunomodulatory impact on human CD4(+) T cells. Blood. 2013;122(7):1182-1191. 


\section{Supplementary materials}



Figure SI Kaplan-Meier analysis of OS according treat.

Note: There was no significant difference in OS between patients in the NACT and non-NACT groups (log-rank test, $P=0.166$ ).

Abbreviations: NACT, neoadjuvant chemotherapy; OS, overall survival.



Figure S2 Kaplan-Meier analysis of OS according TNM.

Note: Patients with GC in stage I/II had longer OS than patients in stage III/IV (logrank test, $P=0.037$ ).

Abbreviations: GC, gastric cancer; NACT, neoadjuvant chemotherapy; OS, overall survival; TNM, tumor, node, metastasis.
OncoTargets and Therapy

\section{Publish your work in this journal}

OncoTargets and Therapy is an international, peer-reviewed, open access journal focusing on the pathological basis of all cancers, potential targets for therapy and treatment protocols employed to improve the management of cancer patients. The journal also focuses on the impact of management programs and new therapeutic agents and protocols on

\section{Dovepress}

patient perspectives such as quality of life, adherence and satisfaction. The manuscript management system is completely online and includes a very quick and fair peer-review system, which is all easy to use. Visit http://www.dovepress.com/testimonials.php to read real quotes from published authors. 